Pacific Journal of Mathematics

ON HILBERT SPACE OPERATORS AND OPERATOR ROOTS 


\title{
ON HILBERT SPACE OPERATORS AND OPERATOR ROOTS OF POLYNOMIALS
}

\author{
S. CATer
}

1. Introduction. The Cayley-Hamilton theorem states that any linear transformation (sometimes called "operator") on a finite dimensional vector space over a field is a root of its characteristic polynomial. On the other hand an operator on an infinite dimensional vector space need not be the root of any nonzero polynomial with scalar coefficients. It is our purpose to give necessary and sufficient conditions for a bounded operator on a complex Hilbert space to be the root of a nonzero polynomial with complex coefficients.

Significant in much linear algebra is the fact that an operator $A$ on a finite dimensional vector space $V$ over an algebraically closed field $F$ must have an eigen value; more precisely, there is a scalar $\lambda$ in $F$ and a nonzero vector $z$ in $V$ such that $(A-\lambda) z=0$. We make the following

DeFinition. An element $\lambda$ in a field $F$ is said to be an eigen value for the operator $A$ on a (possibly infinite dimensional) vector space $V$ over $F$ if there exists at least one nonzero vector $z$ in $V$ such that $(A-\lambda) z=0$. An operator $A$ on $V$ is said to be an eigen value producing (henceforth abbreviated "evp") operator if for each linear maniforld $V^{\prime}$ reducing $A$ and $\neq V$, the operator $A^{\prime}$ induced by $A$ on the quotient space $V / V^{\prime}$ has at least one eigen value.

In particular if $A$ is evp it has an eigen value, because (0) reduces $A$. One example of an evp operator is any operator on a finite dimensional vector space over an algebraically closed field. The central result of the present paper is that a bounded operator on a complex Hilbert space is evp if and only if it is the root of a nonzero polynomial with complex coefficients. Before we introduce Hilbert space operators we establish some algebraic machinery.

2. The structure of evp operators. In this section let $A$ be an operator on a vector space $V$ over the field $F$, and let $V_{\lambda}$ be the linear manifold consisting of all vectors annihilated by some power of the operator $A-\lambda$, for each $\lambda$ in $F$.

Lemma 1. Let $\lambda$ and $\mu$ be distinct scalars and let $z$ be a vector such that

Received March 22, 1961, and in revised form April 25, 1961. 


$$
(A-\lambda)^{e+1}=0 \neq(A-\lambda)^{e} z .
$$

Then

$$
(A-\lambda)^{e+1}(A-\mu) z=0 \neq(A-\lambda)^{e}(A-\mu) z .
$$

In particular if $\mu_{1}, \cdots, \mu_{n}$ is a finite set of scalars different from $\lambda$, then

$$
(A-\lambda)^{e+1} w=0 \neq(A-\lambda)^{e} w
$$

where $w=\left(A-\mu_{1}\right) \cdots\left(A-\mu_{n}\right) z$.

Proof. Since $A$ commutes with itself

$$
(A-\lambda)^{e+1}(A-\mu) z=(A-\mu)(A-\lambda)^{e+1} z=0 .
$$

On the other hand $(A-\lambda)^{e}(A-\mu) z=(A-\mu)(A-\lambda)^{e} z$

$$
\begin{aligned}
& =(A-\lambda)^{e+1} z+(\lambda-\mu)(A-\lambda)^{e} z \\
& =(\lambda-\mu)(A-\lambda)^{e} z \neq 0
\end{aligned}
$$

because $\lambda \neq \mu$. The final statement follows by induction on $n$.

Lemma 2. The $V_{\lambda}$ are linearly independent.

Proof. Suppose $V_{\lambda}$ contains a vector $z$ which is also in the subspace spanned by $V_{\mu_{1}}, \cdots, V_{\mu_{n}}$ where $\mu_{i} \neq \lambda$. Say $z=z_{1}+\cdots+z_{n}$ where $z_{i}$ is in $V_{\mu_{i}}$ and $\left(A-\mu_{i}\right)^{e_{i}} z_{i}=0$. Then

$$
\left(A-\mu_{1}\right)^{e_{1}} \cdots\left(A-\mu_{n}\right)^{e_{n}} z=\left(A-\mu_{1}\right)^{e_{1}} \cdots\left(A-\mu_{n}\right)^{e_{n}}\left(z_{1}+\cdots+z_{n}\right)=0 .
$$

By Lemma $1, z=0$.

Lemma 3. The $V_{\lambda}$ together span $V$ if and only if $A$ is evp.

Proof. Assume the $V_{\lambda}$ span $V$. Let $V^{\prime}$ be a subspace of $V$ reducing $A$ and $\neq V$, and select $z$ in $V-V^{\prime}$. Then $z=z_{1}+\cdots+z_{n}$ where $z_{i}$ is in $V_{\lambda_{i}}$ and consequently there are scalars $\mu_{1}, \cdots, \mu_{m}$ such that $\left(A-\mu_{1}\right) \cdots$ $\left(A-\mu_{m}\right) z=0$. Among the vectors

$$
z,\left(A-\mu_{m}\right) z,\left(A-\mu_{m-1}\right)\left(A-\mu_{m}\right) z, \cdots,\left(A-\mu_{1}\right) \cdots\left(A-\mu_{m}\right) z=0
$$

the first is not in $V^{\prime}$ but the last is in $V^{\prime}$. Let $w$ be the last vector listed which is not in $V^{\prime}$. Obviously there is a scalar $\mu$ such that $(A-\mu) w$ is in $V^{\prime}$. It is clear that $A^{\prime}$, induced by $A$ on $V / V^{\prime}$, has the eigen value $\mu$. Hence $A$ is evp.

Assume $A$ is evp and let $V^{\prime}$ be the subspace spanned by the $V_{\lambda}$. We will show $V^{\prime}=V$ by contradiction; suppose $V \neq V^{\prime}$. Because $A$ is evp 
there exists a scalar $\lambda$ and a vector $z$ in $V-V^{\prime}$ such that $(A-\lambda) z$ is in $V^{\prime}$. Say $\mu_{1}, \cdots, \mu_{m}$ are scalars such that $\left(A-\mu_{1}\right) \cdots\left(A-\mu_{m}\right)(A-\lambda) z=0$. Clearly $\left[\pi_{\mu_{i \neq \lambda}}\left(A-\mu_{i}\right)\right] z$ is a vector which is annihilated by some power of $A-\lambda$; it is in $V_{\lambda}$. Now let $\lambda_{1}, \cdots, \lambda_{n}$ be those $\mu_{i} \neq \lambda$. We have

$$
\left(A-\lambda_{1}\right) z=(A-\lambda) z+\left(\lambda-\lambda_{1}\right) z
$$

and

$$
\left(A-\lambda_{2}\right)\left(A-\lambda_{1}\right) z=\left(A-\lambda_{2}\right)(A-\lambda) z+\left(\lambda-\lambda_{1}\right)(A-\lambda) z+\left(\lambda-\lambda_{2}\right)\left(\lambda-\lambda_{1}\right) z .
$$

Recalling that $V^{\prime}$ reduces $A$ and repeating this argument we see finally that $\left[\pi_{j}\left(A-\lambda_{j}\right)\right] z=c z+w$ where $w$ is in $V^{\prime}$ and $c$ is the scalar $\pi_{j}\left(\lambda-\lambda_{j}\right)$. Since $c \neq 0$ and $c z+w$ is in $V^{\prime}, z$ must be in $V^{\prime}$ contrary to the choice of $z$.

Thus $A$ is evp if and only if $V$ is the direct sum of all the $V_{\lambda}$. Of course in this general context infinitely many $V_{\lambda}$ might be $\neq(0)$ and $V_{\lambda}$ might not be annihilated by any power of $A-\lambda$. We leave to the reader the construction of examples demonstrating these possibilities.

Lemma 4. If $A$ is evp then for each vector $z$ in $V$ there exists a nonzero polynomial $p$ with coefficients in $F$ (depending perhaps on $z$ ) such that $p(A) z=0$.

Proof. Assume $A$ is evp and select $z$ in $V$. By Lemma 3, $z$ is in the subspace spanned by the $V_{\lambda}$ and there exist scalars $\lambda_{1}, \cdots, \lambda_{n}$ such that $\left(A-\lambda_{1}\right)\left(A-\lambda_{2}\right) \cdots\left(A-\lambda_{n}\right) z=0$. Then $\left(x-\lambda_{1}\right) \cdots\left(x-\lambda_{n}\right)$ is an appropriate polynomial.

Lemma 5. Let $F$ be algebraically closed and suppose for each vector $z$ in $V$ there exists a nonzero polynomial $p$ with coefficients in $F$ such that $p(A) z=0$. Then $A$ is evp on $V$.

Proof. Let $V^{\prime}$ be a subspace of $V$ reducing $A$ and $V^{\prime} \neq V$. Select any $z$ in $V-V^{\prime}$. Let $p$ be a polynomial such that $p(A) z=0$. Since $F$ is algebraically closed there exist scalars $c, \lambda_{1}, \cdots, \lambda_{n}$ such that

$$
p(A)=c\left(A-\lambda_{1}\right) \cdots\left(A-\lambda_{n}\right)
$$

and

$$
\left(A-\lambda_{1}\right) \cdots\left(A-\lambda_{n}\right) z=0 .
$$

By the argument employed on the vectors

$$
z,\left(A-\lambda_{1}\right) z,\left(A-\lambda_{1}\right)\left(A-\lambda_{2}\right) z, \cdots,\left(A-\lambda_{1}\right) \cdots\left(A-\lambda_{n}\right) z
$$

in Lemma 3 , it follows that $A$ is evp. 
3. Evp operators on Hilbert space. We now have all the algebraic machinery necessary to tackle the structure of bounded evp operators on complex Hilbert space. Until further notice assume that $A$ is a bounded evp operator on the complex Hilbert space $h$.

Lemma 6. For each scalar $\lambda$ there exists an integer $N$ such that $(A-\lambda)^{N} V_{\lambda}=(0)$.

Proof. Our proof is by contradiction; suppose there exists no integer $N$ such that $(A-\lambda)^{N} V_{\lambda}=(0)$. Let $Z_{n}$ be the null space of the operator $(A-\lambda)^{n}$. Then each $Z_{n}$ is a closed subspace of $h$ and together the $Z_{n}$ span $V_{\lambda}$. The inclusions

$$
Z_{1} \subset Z_{2} \subset Z_{3} \subset \cdots
$$

are all proper, for if $Z_{n}=Z_{n+1}$ for some $n$ every vector annihilated by $(A-\lambda)^{n+1}$ is annihilated by $(A-\lambda)^{n}$ and likewise every vector annihilated by $(A-\lambda)^{m}, m>n$, is annihilated by $(A-\lambda)^{n}$ contrary to assumption. Setting $Z_{0}=(0)$ select a unit vector $z_{n}$ in $Z_{n} \cap Z_{n-1}^{\perp}$ for each index $n>0$ and put $z=\sum_{1}^{\infty} 2^{-n^{2}} z_{n}$. By Lemma 4 there is a nonzero polynomial $p$ with complex coefficients such that $p(A) z=0$. For each index $n>0$ we have $(A-\lambda) Z_{n} \subset Z_{n-1}$. For each index $m$

$$
\begin{aligned}
0 & =\left(p(A) z, z_{m}\right) \\
& =\left((p(A)-p(\lambda)) z, z_{m}\right)+p(\lambda)\left(z, z_{m}\right) \\
& =2^{-m^{2}}\left([p(A)-p(\lambda)]\left[\sum_{n=m+1}^{\infty} 2^{m^{2}-n^{2}} z_{n}\right], z_{m}\right)+2^{-m^{2}} p(\lambda)
\end{aligned}
$$

because $p(A)-p(\lambda)$ contains a factor of $A-\lambda$ and $z_{m}$ is orthogonal to $[p(A)-p(\lambda)]\left[\sum_{n=1}^{m} 2^{-n^{2}} z_{n}\right]$. Dividing out $2^{-m^{2}}$ we obtain

$$
|p(\lambda)| \leqq 2^{-2 m}\|p(A)-p(\lambda)\|
$$

for and $m$, and clearly $p(\lambda)=0$; indeed if $q$ is a polynomial such that $q(\lambda) \neq 0$, then $\left(q(A) z, z_{m}\right) \neq 0$ for infinitely many indices $m$. Let $q$ be the polynomial such that $p(A)=(A-\lambda)^{e} q(A)$ and $q(\lambda) \neq 0$. Now $0=p(A) z=(A-\lambda)^{e} q(A) z$ and $q(A) z$ is in $Z_{e}$. But by the above argument $\left(q(A) z, z_{m}\right) \neq 0$ for infinitely many indices $m$ and $z_{m} \perp Z_{e}$ for $m>e$, contradiction.

LEMMA 7. There are at most finitely many complex scalars $\lambda$ for which $V_{\lambda} \neq(0)$.

Proof. Our proof is by contradiction; suppose there are infinitely many $\lambda$ for which $V_{\lambda} \neq(0)$. Since $A$ is bounded, the set of such $\lambda$ is bounded. Let $\left\{\lambda_{n}\right\}$ be a sequence of distinct members of this set con- 
verging to a fixed scalar $c$ such that $\lambda_{n} \neq c$ for all $n$. Let $N$ be an integer such that $(A-c)^{N} V_{c}=(0)$. (It makes no difference if $V_{c}=(0)$ already.) Let $Z_{n}$ be the null space of the operator $(A-c)^{N}\left(A-\lambda_{1}\right) \cdots\left(A-\lambda_{n}\right)$ for $n>0$ and set $Z_{0}=V_{c}$. All the inclusions

$$
Z_{0} \subset Z_{1} \subset Z_{2} \subset \cdots
$$

are proper by Lemma 1 , and $\left(A-\lambda_{n}\right) Z_{n} \subset Z_{n-1}$. Select in each $Z_{n} \cap Z_{n-1}^{\perp}$ a unit vector $z_{n}$ and put $z=\sum_{1}^{\infty} 2^{-n^{2}} z_{n}$. There is a nonzero polynomial $p$ such that $p(A) z=0$. For each index $m$

$$
\begin{aligned}
& 0=\left(p(A) z, z_{m}\right) \\
& =\left(\left[p(A)-p\left(\lambda_{m}\right)\right] z, z_{m}\right)+\left(\left[p\left(\lambda_{m}\right)-p(c)\right] z, z_{m}\right)+\left(p(c) z, z_{m}\right)
\end{aligned}
$$

and

$$
\begin{aligned}
2^{-m^{2}}\left(\left[p(A)-p\left(\lambda_{m}\right)\right][\right. & \left.\left.\sum_{n=m+1}^{\infty} 2^{m^{2}-n^{2}} z_{n}\right], z_{m}\right) \\
& +2^{-m^{2}}\left[p\left(\lambda_{m}\right)-p(c)\right]+2^{-m^{2}} p(c)=0 .
\end{aligned}
$$

Dividing out $2^{-m^{2}}$ we obtain

$$
|p(c)| \leqq 2^{-2 m}|| p(A)-p\left(\lambda_{m}\right) \|+\left|p\left(\lambda_{m}\right)-p(c)\right| .
$$

As $m$ tends to $\infty,\left\|p(A)-p\left(\lambda_{m}\right)\right\|$ tends to $\|p(A)-p(c)\|$ and $\left|p\left(\lambda_{m}\right)-p(c)\right|$ tends to 0 . Clearly $p(c)=0$; indeed if $q$ is a polynomial such that $q(c) \neq 0$, then $\left(q(A) z, z_{m}\right) \neq 0$ for infinitely many indices $m$. Let $q$ be the polynomial such that $p(A)=(A-c)^{e} q(A)$ and $q(c) \neq 0$. Then $0=p(A) z=(A-c)^{e} q(A) z$ and $q(A) z$ is in $V_{c}=Z_{0}$. By the above argument $\left(q(A) z, z_{m}\right) \neq 0$ for infinitely many $m$ but $z_{m} \perp Z_{0}$ for all $m>0$, contradiction.

We are now able to prove our theorem. Let $A$ be a bounded operator on the complex Hilbert space $h$ and drop the assumption that $A$ is evp.

THEOREM. The following properties are equivalent for $A$.

(1) $A$ is evp.

(2) There is a nonzero polynomial $P$ with complex coefficients such that $P\left(A^{\prime}\right)=0$ on $h$.

Proof. Assume (1). By our development $h$ is the direct sum of $V_{\lambda_{1}}, \cdots, V_{\lambda_{n}}$ and for each index $i=1, \cdots, n$, there is an integer $e_{i}$ such that $\left(A-\lambda_{i}\right)^{e_{i}} V_{\lambda_{i}}=(0)$. Clearly $\pi_{1}^{n}\left(A-\lambda_{i}\right)^{e_{i}}=0$ on $h$ and $\pi_{1}^{n}\left(x=\lambda_{i}\right)^{e_{i}}$ is an appropriate polynomial $P$.

Now assume (2). By Lemma 5, $A$ is evp, and the proof is complete.

The next corollary is of particular interest because it states that a pointwise property is equivalent to a global property. The proof is an immediate consequence of Lemma 5 and our theorem. 
COROLlaRY 1. The following properties are equivalent for $A$.

(1) For each vector $z$ in $h$ there exists a nonzero polynomial $p$ (depending perhaps on $z$ ) such that $p(A) z=0$.

(2) There exists a nonzero polynomial $P$ such that $P(A)=0$ on $h$.

Any operator on a finite dimensional complex Hilbert space is of course evp. Such an operator $A$ must also be bounded and the polynomials in $A$ must constitute a uniformly closed subset of the ring of all operators; the latter fact is due to the theorem that any linear manifold in a finite dimensional normed linear space is closed in norm. Consequently we should not be surprised by

CoRollaRy 2. Let $A$ be a bounded operator on a complex Hilbert space $h$. Then the following properties are equivalent for $A$.

(1) $A$ is evp.

(2) the polynomials in A constitute a uniformly closed subset of $L(h)$, the ring of all bounded operators on $h$.

Proof. First suppose $A$ is evp. Let $P$ be a nonzero polynomial of minimal degree such that $P(A)=0$ and say $n$ is the degree of $P$. Then any polynomial in $A$ is equal to an appropriate polynomial in $A$ of degree less than $n$. It follows that the polynomials in $A$ form a finite dimensional linear manifold in the normed linear space $L(h)$ over the complex field, and this manifold must be closed in norm.

Now suppose the polynomials in $A$ form a uniformly closed subset of $L(h)$. (The author is indebted to $\mathrm{B}$. Yood for the remainder of the argument.) Select a positive scalar $r$ greater than the spectral radius of $A$. Then $A-r$ is nonsingular and $(r-A)^{-1}$ is the limit of a convergent series in $A$ and this series must converge to a polynomial $p(A)$. Thus $(r-A) p(A)=1$ and $A$ is a root of the nonzero polynomial $(r-x) p(x)-1$.

4. Principal idempotents. Given operators $A$ and $B$ on the respective Hilbert spaces $h$ and $k$ we say $A$ is similar to $B$ if there exists an invertible linear tranformation $S$ of $h$ onto $k$ such that $S$ and $S^{-1}$ are bounded and $S A S^{-1}=B$ on $k$. This section concerns the theorem on principal idempotents on finite dimensional vector spaces (see [7], pp. 175-7) which states that a matrix is similar to a diagonal matrix if and only if it is a linear combination of mutually orthogonal idempotent matrices (of course no condition of boundedness enters here). In particular if the field of scalars is the complex field this theorem remains valid when "normal matrix" replaces "diagonal matrix".

The following corollary generalizes this result by means of bounded normal evp operators. By the spectral mapping theorem all the spectral 
values of a bounded normal evp operator are roots of a certain nonzero polynomial; hence such an operator $N$ has finite spectrum, and furthermore by the spectral theorem all of its spectral values are eigen values and $N$ is a linear combination of finitely many mutually orthogonal projections the sum of which is the identity.

CoRollaRy 3. For an operator A mapping $h$ into $h$ the following are equivalent.

(1) $A$ is similar to a bounded normal evp operator $N$ on a Hilbert space.

(2) There exist a finite collection $E_{1}, \cdots, E_{n}$ of mutually orthogonal nonzero idempotent operators, each mapping $h$ onto a closed subspace of $h$, and distinct scalars $\lambda_{1}, \cdots, \lambda_{n}$ such that $I=\sum_{1}^{n} E_{i}$ and $A=\sum_{1}^{n} \lambda_{i} E_{i}$.

Proof. Assume (1). Then $N$ is a linear combination of mutually orthogonal nonzero projections $P_{1}, \cdots, P_{n}$; say $N=\sum_{1}^{n} \lambda_{i} P_{i}$ and $I=\sum_{1}^{n} P_{i}$ where $\lambda_{i} \neq \lambda_{j}$ if $i \neq j$. There exists an invertible linear transformation $S$ such that $S A S^{-1}=N$ and

$$
A=S^{-1} N S=S^{-1}\left(\sum_{1}^{n}{ }_{1}^{n} \lambda_{i} P_{i}\right) S=\sum_{1}^{n}{ }_{1}^{n} \lambda_{i} S^{-1} P_{i} S,
$$

and obviously $E_{i}=S^{-1} P_{i} S$ suffices in (2). Of course the operators $A$, $E_{1}, \cdots, E_{n}$ are bounded because $S, S^{-1}, N, P_{1}, \cdots, P_{n}$ are bounded. Furthermore each linear manifold $E_{i} h$ is closed in $h$ because the range of a bounded idempotent operator on Hilbert space must be closed.

Now assume (2). Then each $E_{i} h$ is a closed linear subspace of $h$ and $h$ is the direct linear sum of the $E_{i} h$. Let $k$ be the direct orthogonal product of the Hilbert spaces $E_{i} h$. Let $S_{i}$ be the linear transformation mapping $h$ onto $E_{i} h$ (regarded as a subspace of $k$ ) which is the identity on $E_{i} h$ and which annihilates every vector in $E_{j} h, j \neq i$. Then $S=\sum_{1}^{n} S_{i}$ is an invertible linear transformation of $h$ onto $k$. It suffices to show that $S$ and $S^{-1}$ are bounded; for if they are, the operators $S E_{i} S^{-1}$ are mutually orthogonal projections on $k$ and $A$ is similar to the bounded normal evp operator $S A S^{-1}=\sum_{1}^{n} \lambda_{i} S E_{i} S^{-1}$ on $k$. That $S^{-1}$ is bounded follows immediately form the inequality $\left\|S^{-1} z\right\|^{2} \leqq n_{2}\|z\|^{2}$, all $z$ in $k$. By the closed graph theorem $S$ is bounded also, and the proof is complete.

\section{REFERENCES}

1. G. Birkhoff and S. Maclane, A Survey of Modern Algebra, 1941.

2. J. Dixmier Les Algebres D'operateurs dans L'espace Hiblertien, 1957.

3. P. R. Halmos, Finite Dimensional Vector Spaces, 1958.

4. , Introduction to Hilbert Space, 1951. 
5. M. Jacobson, Lectures in Abstract Algebra, vol. II, 1953.

6. N. H. McCoy, Introduction to Modern Algebra, 1960.

7. S. Perlis, Theory of Matrices, 1952.

8. M. H. Stone, Linear Transformations on Hilbert Space, 1932.

UNIVERSITY OF OREGON 


\title{
PACIFIC JOURNAL OF MATHEMATICS
}

\author{
EDITORS
}

Ralph S. Phillips

Stanford University

Stanford, California

M. G. Arsove

University of Washington

Seattle 5, Washington
A. L. Whiteman

University of Southern California Los Angeles 7, California

Lowell J. Paige

University of California

Los Angeles 24, California

\section{ASSOCIATE EDITORS}
E. F. BECKENBACH
D. DERRY
M. OHTSUKA
H. L. ROYDEN
E. SPANIER
E. G. STRAUS
T. M. CHERRY
F. WOLF

\section{SUPPORTING INSTITUTIONS}

\author{
UNIVERSITY OF BRITISH COLUMBIA \\ CALIFORNIA INSTITUTE OF TECHNOLOGY \\ UNIVERSITY OF CALIFORNIA \\ MONTANA STATE UNIVERSITY \\ UNIVERSITY OF NEVADA \\ NEW MEXICO STATE UNIVERSITY \\ OREGON STATE UNIVERSITY \\ UNIVERSITY OF OREGON \\ OSAKA UNIVERSITY \\ UNIVERSITY OF SOUTHERN CALIFORNIA
}

\author{
STANFORD UNIVERSITY \\ UNIVERSITY OF TOKYO \\ UNIVERSITY OF UTAH \\ WASHINGTON STATE UNIVERSITY \\ UNIVERSITY OF WASHINGTON \\ $*$
AMERICAN MATHEMATICAL SOCIETY \\ CALIFORNIA RESEARCH CORPORATION \\ SPACE TECHNOLOGY LABORATORIES \\ NAVAL ORDNANCE TEST STATION
}

Mathematical papers intended for publication in the Pacific Journal of Mathematics should be typewritten (double spaced), and the author should keep a complete copy. Manuscripts may be sent to any one of the four editors. All other communications to the editors should be addressed to the managing editor, L. J. Paige at the University of California, Los Angeles 24, California.

50 reprints per author of each article are furnished free of charge; additional copies may be obtained at cost in multiples of 50 .

The Pacific Journal of Mathematics is published quarterly, in March, June, September, and December. Effective with Volume 13 the price per volume (4 numbers) is $\$ 18.00$; single issues, $\$ 5.00$. Special price for current issues to individual faculty members of supporting institutions and to individual members of the American Mathematical Society: $\$ 8.00$ per volume; single issues $\$ 2.50$. Back numbers are available.

Subscriptions, orders for back numbers, and changes of address should be sent to Pacific Journal of Mathematics, 103 Highland Boulevard, Berkeley 8, California.

Printed at Kokusai Bunken Insatsusha (International Academic Printing Co., Ltd.), No. 6, 2-chome, Fujimi-cho, Chiyoda-ku, Tokyo, Japan.

PUBLISHED BY PACIFIC JOURNAL OF MATHEMATICS, A NON-PROFIT CORPORATION

The Supporting Institutions listed above contribute to the cost of publication of this Journal, but they are not owners or publishers and have no responsibility for its content or policies. 


\section{Pacific Journal of Mathematics}

\section{Vol. 12, No. $2 \quad$ February, 1962}

William George Bade and Robert S. Freeman, Closed extensions of the Laplace operator determined by a general class of boundary conditions . . . . . . . 395

William Browder and Edwin Spanier, H-spaces and duality ............. 411

Stewart S. Cairns, On permutations induced by linear value functions . . . . . . . 415

Frank Sydney Cater, On Hilbert space operators and operator roots of

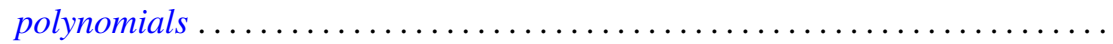

Stephen Urban Chase, Torsion-free modules over $K[x, y] \ldots \ldots \ldots \ldots \ldots \ldots \ldots 437$

Heron S. Collins, Remarks on affine semigroups . . . . . . . . . . . . . . . . 449

Peter Crawley, Direct decompositions with finite dimensional factors . . . . . . . 457

Richard Brian Darst, A continuity property for vector valued measurable

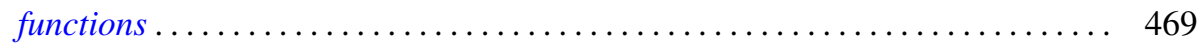

R. P. Dilworth, Abstract commutative ideal theory ................. 481

P. H. Doyle, III and John Gilbert Hocking, Continuously invertible spaces . . . . . . 499

Shaul Foguel, Markov processes with stationary measure . . . . . . . . . . . 505

Andrew Mattei Gleason, The abstract theorem of Cauchy-Weil ............ 511

Allan Brasted Gray, Jr., Normal subgroups of monomial groups . . . . . . . . . . 527

Melvin Henriksen and John Rolfe Isbell, Lattice-ordered rings and function

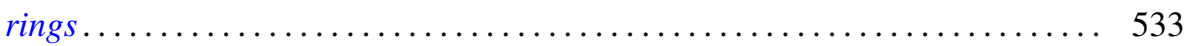

Amnon Jakimovski, Tauberian constants for the $[J, f(x)]$ transformations . ..... 567

Hubert Collings Kennedy, Group membership in semigroups . . . . . . . . . . . 577

Eleanor Killam, The spectrum and the radical in locally $m$-convex algebras ..... 581

Arthur H. Kruse, Completion of mathematical systems . . . . . . . . . . . . . 589

Magnus Lindberg, On two Tauberian remainder theorems ................ 607

Lionello A. Lombardi, A general solution of Tonelli's problem of the calculus of

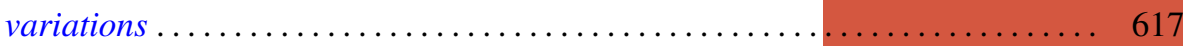

Marvin David Marcus and Morris Newman, The sum of the elements of the powers

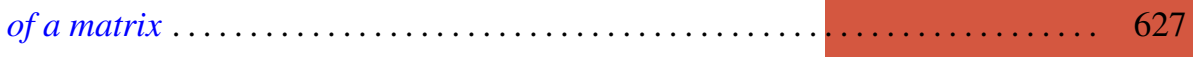

Michael Bahir Maschler, Derivatives of the harmonic measures in

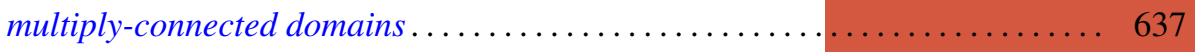

Deane Montgomery and Hans Samelson, On the action of $\mathrm{SO}(3)$ on $S^{n} \ldots \ldots \ldots 649$

J. Barros-Neto, Analytic composition kernels on Lie groups . . . . . . . . . . . . 661

Mario Petrich, Semicharacters of the Cartesian product of two semigroups ...... 679

John Sydney Pym, Idempotent measures on semigroups . . . . . . . . . . . . 685

K. Rogers and Ernst Gabor Straus, A special class of matrices . . . . . . . . . . . . 699

U. Shukla, On the projective cover of a module and related results . . . . . . . . . 709

Don Harrell Tucker, An existence theorem for a Goursat problem . . . . . . . . . . . 719

George Gustave Weill, Reproducing kernels and orthogonal kernels for analytic

differentials on Riemann surfaces ......................... 729

George Gustave Weill, Capacity differentials on open Riemann surfaces ........ 769

G. K. White, Iterations of generalized Euler functions . . . . . . . . . . . . . 777

Adil Mohamed Yaqub, On certain finite rings and ring-logics . . . . . . . . . 785 